\title{
Aportes de experiencias de Educación Superior Rural de Colombia, Brasil y México a la construcción de una propuesta curricular en el Sur del Tolima (Colombia)
}

\footnotetext{
Laura Valentina Amado Ochoa ${ }^{1}$, iD Laura Marcela García Salamanca ${ }^{2}$, iD Jorge Jairo Posada Escobar ${ }^{3}$, iD Luisa Fernanda Ramírez Cuervo ${ }^{4}$

1, 2, 4 Universidad Colegio Mayor de Cundinamarca. Facultad de Ciencias Sociales. Calle 28 \# 5b - 02. Bogotá, Colombia. ${ }^{3}$ Universidad Pedagógica Nacional de Colombia.

Autor para correspondência/Author for correspondence: Ivamado@unicolmayor.edu.co
}

\begin{abstract}
RESUMEN. En este artículo se presenta un estudio documental que tiene por objetivo reconocer los principios, propuestas curriculares y estrategias metodológicas de las experiencias de educación superior rural en Colombia, Brasil y México en los últimos 10 años. El análisis de las experiencias permite generar aportes para una región del centro de Colombia, ya que el estudio se realiza en el marco de la investigación "Construcción participativa de un programa curricular de educación superior rural de base agroecológica como alternativa al desarrollo territorial en el pos-acuerdo". Dichas experiencias analizadas se fundamentan en el reconocimiento de las y los campesinos e indígenas como sujetos culturales, en la necesidad de la formación para la transformación social, la articulación de componentes de formación técnica con lo político y lo ecológico. Las experiencias proponen currículos contextualizados, con estrategias metodológicas y experienciales que parten de los saberes campesinos e indígenas, con el trabajo de proyectos productivos, de servicio a la comunidad e investigativos.
\end{abstract}

Palabras clave: educación superior rural, currículo, estrategias metodológicas, saberes, experiencias. 


\title{
Contributions of Rural Higher Education experiences from Colombia, Brazil and Mexico to the construction of a curriculum proposal in South Tolima (Colombia)
}

\begin{abstract}
This article presents a documentary study that aims to recognize the principles, curricular proposals and methodological strategies of the experiences of rural higher education in Colombia, Brazil and Mexico in the last 10 years. The analysis of the experiences makes it possible to generate contributions for a region of central Colombia, since the study is carried out within the framework of the research "Participatory construction of a curricular program of rural higher education based on agroecology as an alternative to territorial development in the pos -agreement". These experiences analysed are based on the recognition of peasants and indigenous peoples as cultural subjects, on the need for training for social transformation, the articulation of technical training components with the political and ecological aspects. The experiences propose contextualized curricula, with methodological and experiential strategies that start from peasant and indigenous knowledge, with the work of productive projects, community service and research.
\end{abstract}

Keywords: rural higher education, curricula, methodological strategies, knowledge, experiences. 


\section{Contribuições de experiências de Educação Superior Rural da Colômbia, Brasil e México para a construção de uma proposta curricular no Sul de Tolima (Colômbia)}

RESUMO. Este artigo apresenta um estudo documental que visa reconhecer os princípios, propostas curriculares e estratégias metodológicas das experiências de educação superior rural na Colômbia, Brasil e México nos últimos 10 anos. A análise das experiências permite gerar contribuições para uma região do centro da Colômbia, já que o estudo se realiza no marco da pesquisa "A construção participativa de um programa curricular de ensino superior rural baseado na agroecologia como alternativa ao desenvolvimento territorial no pós-acordo". As experiências analisadas baseiam-se no reconhecimento dos camponeses e indígenas como sujeitos culturais, na necessidade de formação para a transformação social, na articulação dos componentes da formação técnica com os aspectos políticos e ecológicos. As experiências propõem currículos contextualizados, com estratégias metodológicas e vivenciais que partem dos saberes camponeses e indígenas, com o trabalho de projetos produtivos, serviço comunitário e pesquisa.

Palavras-chave: educação superior rural, currículo, saberes, estratégias metodológicas, experiências. 


\section{Introducción}

En este artículo se presenta el avance de un estudio documental sobre experiencias de educación superior rural en Colombia y su comparación con dos experiencias reconocidas de América Latina en este campo como son: La Educación del Campo de Brasil y la Universidad de la Tierra de Oaxaca, México. Esta revisión documental se realiza en el marco de la investigación desarrollada por el Sistema Universitario Estatal - Bogotá "Construcción participativa de un programa curricular de educación superior rural de base agroecológica como alternativa al desarrollo territorial en el pos-acuerdo". La investigación se efectúa con las comunidades del Distrito de Riego El Triángulo del Tolima (DRTT), zona localizada en el centro-oeste de Colombia. ${ }^{\mathrm{i}}$

El DRTT encierra los municipios de Coyaima (85 \%), Natagaima (11\%) y Purificación (4\%), sobre un área de 34.143 hectáreas, de las cuales serán irrigadas 20.402. La región es ocupada por comunidades indígenas descendientes de los Pijao y agricultores campesinos, que tienen el riesgo de ser desplazados de ese territorio, dada la presión de inversionistas foráneos que tienen el interés de ocupar dichas tierras irrigadas. El pueblo indígena Pijao vive en el sur del departamento del
Tolima, actualmente cuenta con más de 50 mil habitantes (DANE, 2018). "De los 77.587 habitantes del DRTT, la gran mayoría son campesinos de raza indígena (76,9\% en Coyaima, 47,1\% en Natagaima y $13,4 \%$ en Purificación) con un bajo porcentaje de mestizaje, y de modo general, viven en condiciones de marginación social" (Acevedo, 2013, p. 2).

Se busca con este estudio documental realizar una reflexión que pueda aportar principios, propuestas curriculares, estrategias metodológicas para la construcción de programas educativos y procesos productivos de base agroecológica para la región del DRTT, es por esto que se seleccionaron estas experiencias de educación superior rural teniendo como criterio el que estas se hayan desarrollado en contextos campesinos o indígenas similares en sus características sociales a las del DRTT. Se analizaron documentos sobre las experiencias que han realizado sus prácticas en los últimos diez años porque es un período de tiempo adecuado, es decir experiencias que tuvieran vigencia pero que su trayectoria les permitiera algún impacto en las comunidades rurales.

El acuerdo de paz con las FARC-EP (2016) ha constituido una nueva oportunidad para colocar el tema agrario en el centro del debate político nacional. 
Es en este escenario, que consideramos necesario que la academia colombiana asuma un papel en el desarrollo de diversas iniciativas frente a la problemática agraria como prerrequisito para lograr una paz estable y duradera. En este sentido, se busca con este estudio formular una propuesta curricular de manera participativa, que aporte al desarrollo territorial y a la construcción de paz.

Las comunidades del sur DRTT, como otras zonas rurales del país, han sido afectadas por la violencia política, igualmente en esta zona se manifiestan problemáticas educativas y sociales.

En Colombia el analfabetismo en las zonas rurales sigue siendo alto: "Para la población de 15 y más años, este indicador se ubicó en un $12,1 \%$ en las zonas rurales" (Departamento Nacional de Planeación Colombia - DNP -, 2018, p. 286). En cuanto a la educación media: "La cobertura neta en educación media rural apenas llega a un $31 \%$, de modo que presenta una brecha de 16 puntos porcentuales con respecto a la cobertura urbana para el mismo nivel" (p. 287). Una gran parte de los y las jóvenes no acceden a la educación $\mathrm{y}$ esto se agrava o presenta una gran desigualdad en la educación rural y en algunas regiones del país. Y si esto se cruza por grupos poblaciones, las poblaciones indígenas y las poblaciones afrocolombianas tienen menos oportunidades educativas. Además de las desigualdades en cobertura, también se dan inequidades en relación con la calidad educativa medida en las pruebas Saber y esto es más evidente en la falta de pertinencia de los programas educativos para las diferentes regiones del país y para las zonas rurales (Serna \& Patiño, 2018; Pineda \& Suaza, 2017). Asimismo, en la zona del DRTT hay una alta deserción escolar a nivel rural, la mayor parte de los y las estudiantes que terminan la educación media no siguen estudios de educación superior.

Partimos de considerar que esta es una investigación documental (Páramo, 2011) que da cuenta de un campo de conocimiento sobre la producción realizada en un espacio $\mathrm{y}$ tiempo determinados, las revisiones documentales son ejercicios investigativos sobre otros estudios y reflexiones. Se requiere, por tanto, un trabajo interpretativo hermenéutico.

Para esta investigación se realizó el estudio de estado del arte, según Jiménez (2004), Calvo y Castro (1995), Gómez, Galeano y Jaramillo (2015), los estados del arte posibilitan la sistematización de investigaciones realizadas en un campo temático específico, muchas veces haciendo comparaciones para determinar 
los desarrollos y los vacíos en el conocimiento de dicho campo. Para seleccionar e identificar los documentos se realizó la exploración en bases de datos para artículos como Scopus, Dialnet, Redalyc, Scielo; también se hizo una revisión en Google académico para la búsqueda de libros y en los repositorios de tesis de universidades de Colombia, Brasil y México. Para esta búsqueda se utilizó como descriptores de búsqueda las expresiones: experiencias de educación superior rural, educación superior rural, educación superior campesina, educación universitaria rural.

Para realizar la búsqueda documental, la organización, sistematización y análisis nos basamos en la siguiente pregunta: ¿Qué aspectos de las experiencias en educación superior rural que se han realizado en Colombia, Brasil y México en los últimos 10 años son relevantes para el proceso de construcción de programas educativos y productivos de base agroecológica en el DRTT?

\section{Conceptos claves: principios, propuestas curriculares, estrategias metodológicas}

Nos referimos a principios como los fundamentos teóricos de las experiencias analizadas, estos tienen que ver con las concepciones acerca de los sujetos y las sujetas que se buscan formar, a las concepciones de sociedad que se proponen contribuir a crear con su trabajo educativo, a los fundamentos epistemológicos (sobre el conocimiento, sobre la relación de los y las sujetos con el saber), o a las concepciones metodológicas generales en que fundamentan su propuesta educativa, a los autores y autoras que los inspiran, a las teorías o modelos pedagógicos generales en los que inscriben sus propuestas educativas (Flórez, 1994).

Los principios también incluyen, en el caso específico de la educación rural, las ideas acerca de la ruralidad, del campo, de los y las campesinas, a la lectura que se hace de las problemáticas de la ruralidad y la educación rural. Los principios de una experiencia educativa pueden ser disciplinares, interdisciplinares, sociopolíticos, filosóficos y/o pedagógicos.

Cuando hablamos de propuestas curriculares tenemos que referirnos al concepto de currículo: "es un término que genera mucha controversia, su significado tiene un carácter polisémico". (Caicedo \& Calderón, 2016, p. 58). A pesar de las discusiones sobre este concepto, en relación al currículo, nosotros acogemos las ideas de Magendzo (1986) el cual considera que es un proceso de selección cultural de los contenidos y objetivos, no es obra del azar, ni una actividad inocente, sino que está influido por las perspectivas 
y valores de quienes realizan este proceso.

De manera similar, De Alba, 1998, entiende por currículo: “... la síntesis de elementos culturales (conocimientos, valores, costumbres, creencias, hábitos) que conforman una propuesta políticoeducativa pensada e impulsada por diversos grupos y sectores sociales cuyos intereses son diversos y contradictorios..." (p. 59). En la misma dirección, pensamos el currículo como política cultural, porque “el currículo es el resultado de una selección de cultura y es un campo conflictivo de producción cultural, de enfrentamientos entre sujetos, conceptos de conocimiento, formas de entender $\mathrm{y}$ construir el mundo" (Lopes \& Macedo, 2011, p. 111). Silva (2006), considera que esta selección cultural es política, se constituye en un espacio donde "se concentran y se desdoblan las luchas en torno de los diferentes significados sobre lo social y sobre lo político" (p. 10). Desde esta perspectiva se asume el currículo, retomando los autores anteriores, como una selección cultural, como una política cultural que refleja unos determinados intereses, unas convicciones y lecturas del mundo.

En el análisis de las experiencias de educación rural superior, entendemos el currículo como un proyecto que se vuelca a la formación de sujetos en situaciones históricas y sociales a fin de responder a las necesidades educativas de las personas, de los grupos sociales $y$ de las comunidades. Cuando indagamos por el currículo nos preguntamos por qué saberes (selección cultural), conocimientos, habilidades, competencias, valores se buscan enseñar y aprender y cómo están organizados y estructurados. Para el análisis y construcción de propuestas de educación superior rural (sobre todo en comunidades indígenas) se ve necesario retomar los aportes críticos de Katerin Arias (2016), la cual considera que la cual considera fundamental la construcción participativa de conocimientos con las comunidades y así reconocer los saberes de los pueblos indígenas tradicionalmente excluidos del currículo escolar.

En relación a las estrategias metodológicas, García y Cañal (1995) señalan las limitaciones y ambigüedades del concepto de metodologías, el cual se ha definido de manera etérea y de forma polisémica. Los autores definen la estrategia metodológica como: “... un sistema peculiar constituido por unos determinados tipos de enseñanza la cual está definida por los tipos de actividades que incluye y por el esquema organizativo que regulan relaciones entre las actividades" (p. 6). De igual manera, para Londoño y Calvache (2010) una estrategia 
metodológica es una actividad conscientemente planeada para lograr un fin, o como un "plan que integra la sucesión de acciones de una organización en un todo coherente" (p. 24). En el análisis de las experiencias asumimos las estrategias metodológicas como las concepciones generales y las propuestas acerca de cómo se busca que los y las estudiantes o participantes en los procesos educativos logren aprendizajes, puedan intercambiar saberes, conocimientos y también mejoren las habilidades, se fortalezcan y construyan valores; es, por tanto, el conjunto de actividades que sirven para propiciar el aprendizaje de los participantes en los procesos de formación.

Las metodologías no se reducen a asuntos técnicos o recetas, sino que estas se derivan de los fundamentos pedagógicos y teóricos de propuestas pedagógicas y educativas; entre estos fundamentos por lo general están unos propósitos generales, una visión de los sujetos y las sujetas que se contribuye a formar, de las concepciones acerca de la realidad social, de los saberes y de los conocimientos que se buscan generar o ayudar a construir. También estas metodologías dependen de los contextos educativos, de las etapas de los procesos educativos, y de las interacciones que se buscan generar.

\section{Experiencias de Educación Superior Rural}

A continuación, se analizan cinco experiencias de educación superior rural que se han llevado a cabo a nivel internacional y nacional (Colombia), las cuales permiten una mirada amplia de las construcciones curriculares desde énfasis territoriales y culturales de las comunidades a quienes se dirigen dichas propuestas.

\section{La Experiencia de la Educación del Campo de Brasil}

Las experiencias de Educación del Campo de Brasil, así como las Licenciaturas para la formación de Educadores del Campo, nacen como fruto de las luchas sociales por los derechos de los campesinos, por el derecho a la tierra y por el derecho a la educación, en especial las luchas del movimiento, Sin Tierra, así lo demuestran diferentes estudios y publicaciones (Bicalho, 2017; Barbosa, 2014; Caldart, 2003, 2012; Molina \& Sá, 2015, entre otros).

Para Barbosa, la creación de la Educación del Campo es resultado de una lucha social, pero también epistémicopolítica. "La Educación del Campo... es una concepción de educación vinculada al conjunto de saberes, a la cultura y a la 
identidad de los pueblos del campo". (Barbosa, 2014, p. 1).

Una de las inspiraciones de la Educación del Campo es la experiencia del pedagogo soviético Pistrak, el cual propone la vinculación de la educación a los procesos sociales vividos y a la transformación social; se busca articular la educación con la vida del campo. En este sentido, esta concepción de la escuela del campo destaca el reconocimiento de los y las campesinos como sujetos de cultura, pero también se plantea como una educación politécnica, donde se aprenden los diferentes campos técnicos, para que la juventud rural pueda acceder a los derechos de cualquier ciudadano de la sociedad global y, al mismo tiempo, tener alternativas para quedarse en el campo.

Molina y Sá (2015) exponen lo siguiente acerca de los fundamentos de la licenciatura de Educación del campo:

... se considera la formación escolar como una de las dimensiones del proceso educativo, se pretende formar educadores capaces de promover una articulación profunda entre escuela y comunidad. Por tanto, uno de los principios cultivados con más fuerza se refiere a la construcción de las habilidades necesarias para que estos futuros educadores puedan internalizar las condiciones de comprensión de las relaciones de la escuela con la vida. (p. 136).
Freitas (2010) considera que lo importante es situar la educación en perspectiva de transformación social, por esto, la Educación del Campo busca cambiar la forma de producir conocimiento, rechazando la exclusión y subordinación social.

Con base en Pistrak y con la orientación de Luiz Carlos Freitas, la experiencia de la licenciatura en Educación del Campo de la Universidad de Brasilia ha adoptado como ideas claves: "una matriz educativa multidimensional, que permita ampliar la función social de la escuela; el diálogo con las agencias formadoras del medio; el trabajo y el desarrollo humano integral como base del aprendizaje...". (Molina \& Sá, 2015, p. 138).

La licenciatura tiene una propuesta curricular estructurada (Educación Formal Superior). Constituye un currículo integrado e interdisciplinario (articulación de disciplinas y áreas del saber), está organizado en tres Núcleos: Núcleo Básico, Núcleo de Estudios Específicos y Núcleo de actividades integradoras.

- Núcleo de Estudios Básicos: Economía Política; Filosofía; Políticas Educativas; Teoría Pedagógica; Lectura, Producción e Interpretación de Textos.

- Núcleo de Estudios Específicos: Eje 1 - Docencia por área de Conocimiento: Lenguajes (Lingüística, Artes y Literatura); Ciencias de la Naturaleza y Matemática. - Eje 2 Gestión de 
procesos educativos escolares. - Eje 3 Gestión de procesos educativos comunitarios.

- Núcleo de Actividades Integradoras: Prácticas Pedagógicas, Investigación, Pasantías, Seminarios Integradores, Otras Actividades Científico-Culturales. (Molina \& Sá, 2015, pp. 134-135).

Su estructuración se basa en la teoría de los complejos de estudio elaborada por Pistrak, conjunto de múltiples relaciones por las que la realidad es integrada. Dentro de esta orientación, la construcción de los complejos de estudio, o de enseñanza, como lo plantea Pistrak, son una metodología que se emplea en la Licenciatura en Educación del Campo, buscando establecer la conexión entre la escuela y la vida, preocupándose por interpretar e intervenir en la realidad (Freitas, 2010). Diversas disciplinas pueden usar un complejo como escenario para desarrollar sus conceptos. Lo importante es garantizar la unidad teoríapráctica. El trabajo se considera como el principio general educativo de inserción en la realidad, incluyendo, además del trabajo productivo, se propone la prestación de servicios en espacios colectivos. Articulada a la dimensión del trabajo como principio educativo, se encuentra la formación para la autodirección o auto organización (Molina \& Sá, 2015).

De esta experiencia resaltamos su vinculación con la lucha por la tierra desde el movimiento campesino y su estrategia metodológica de alternancia entre tiempo de formación en la universidad y tiempo de formación en la comunidad, lo que permite vincular teoría y práctica y generar conocimientos más cercanos a las comunidades campesinas.

\section{La Universidad de la Tierra (Unitierra) de Oaxaca (México)}

Según Ezpeleta y Weis (1996) en México los y las jóvenes estudiaban en escuelas rurales donde sólo se enseñaban materias básicas y si deseaban continuar con sus estudios, no contaban con una posibilidad o alternativa para continuar con los mismos. Así mismo, la gestión escolar en zonas rurales de extrema pobreza no ha tenido en cuenta las expectativas de escolaridad de las comunidades, ni la precariedad institucional de las escuelas.

A raíz de esta problemática, y como respuesta a dicha preocupación, se creó Unitierra, ubicada en Oaxaca, y con una sede adicional en San Cristóbal de Las Casas, a través de la coalición de organizaciones indígenas y no indígenas en el 2001 (Esteva, 2014). Asimismo, para su creación fue importante la experiencia pedagógica de los y las fundadoras, la participación en múltiples reflexiones contribuyó a entender que las cuestiones ambientales y ecológicas no deben 
separarse de las cuestiones sociales y políticas.

Unitierra tiene el propósito principal de ser un espacio autónomo y abierto en donde los y las jóvenes que tengan el interés de estudiar puedan hacerlo sin la necesidad de un título escolar en los campos de su interés y así aprender haciendo. Lo anterior, es un paradigma que posibilita una educación abierta y nada condicionada a un certificado principalmente-, lo que conlleva a una concepción participativa y adaptada a las condiciones de los contextos rurales, en donde hay bajos índices de educación básica y secundaria. Así mismo, mantiene su enfoque hacia la educación para la vida y potenciación de las capacidades de los y las jóvenes, tal como lo expresa Esteva (2014) quien crítica la educación actual, la cual considera que el proceso de aprendizaje es una actividad que no se relaciona con las actividades de la vida. Para Esteva la educación debe estar en relación con el momento histórico, y requiere propiciar la capacidad de los sujetos de pensar.

Para ingresar a Unitierra como estudiante las personas solo deben contar con una visión clara frente a su vida y qué proyectos quieren a futuro, ya que el desarrollo de estos es fundamental frente al enfoque que la universidad tiene de sus estudiantes, es decir que para ser parte de Unitierra no es necesario cumplir con ningún requerimiento. La universidad pretende crear las capacidades para que la gente sea capaz de hacer en vez de consumir (Oster-Katz, 2005).

Esteva (2014) expresa los términos en los que se fundamenta el objetivo y función de esta universidad, primero el término aprendizaje es entendido como un ámbito de la vida cotidiana; en segundo lugar, la investigación se comprende bajo la reflexión de la acción por medio de un diálogo intercultural; y, por último, el estudio es entendido como un ejercicio autónomo. Unitierra se fundamenta en el pensamiento de Ivan Illich el cual no considera que, al estudio como medio escalar en la pirámide de los cursos académicos, títulos, certificados, entre otros; es por esto que en la universidad de la Tierra se aprende sin profesores, currículos o libros (Zaldivar, 2009).

Frente a la propuesta curricular manejada en Unitierra, Esteva (2014) menciona que no existen jerarquías formales o profesionales, teniendo en cuenta esto, los programas y talleres están dirigidos para estudiantes, profesores e investigadores nacionales 0 internacionales. Para cualquier persona que esté interesada, Unitierra organiza programas específicos y estancias de 
actividad que incluyen ciclos de estudio en sus instalaciones y visitas a experiencias o estancias en comunidades. Las estrategias metodológicas de Unitierra ponen énfasis en lo siguiente: Aprender del mundo al convertirse en aprendices de las personas que se dedican a la actividad de interés, aprender a transformar la realidad de sus propias comunidades y territorios de origen, el aprendizaje debe ir en concordancia con la persona que está aprendiendo, a su ritmo y manera de trabajar a aprender, aprender a aprender por uno mismo a partir de los métodos de aprendizaje necesarios, y aprender a aprender con otras personas desde diferentes métodos como círculos de estudio, talleres o conferencias (Esteva, 2014). Los contenidos que se enseñan en Unitierra son flexibles y se centran en el desarrollo de capacidades para aprender a investigar y transformar las realidades de las comunidades rurales de acuerdo a los intereses de los y las estudiantes.

La singularidad de Unitierra se enfoca bajo el principio de que todos $\mathrm{y}$ todas tienen algo que aprender de las demás personas, todas y todos tienen algún conocimiento para ofrecer. En esta experiencia se genera la posibilidad de aprender sin la necesidad de títulos, certificados académicos o un currículo establecido; se organiza y apoya a los y las estudiantes para el desarrollo de proyectos personales y colectivos de aprendizaje.

\section{El Proyecto Utopía de la Universidad de la Salle (Colombia)}

La Universidad de la Salle empezó el proyecto Utopía desde el 2008 como una alternativa que permite a los y las jóvenes de zonas rurales del país tener oportunidades educativas y productivas acordes con sus necesidades y las de sus territorios para lograr la transformación social, política, cultural y productiva de las zonas. Es por esto que los objetivos del programa son convertir a los y las jóvenes bachilleres de zonas rurales afectadas por la exclusión y la violencia en ingenieros agrónomos con la metodología "aprender haciendo y enseñar demostrando", que permita la transformación social, política y productiva del país. Así, se promueve la implementación de los proyectos productivos de los y las jóvenes, como resultado del proceso (Universidad de la Salle, 2020).

El proyecto está estructurado en 12 cuatrimestres en los que los y las estudiantes aprenderán los conocimientos necesarios bajo las 4 áreas de formación reflejados en la malla curricular, a partir del enfoque de aprender haciendo $\mathrm{y}$ aprendizaje a partir de la experiencia. 
El área fundamentadora abarca las bases teóricas que le son propias al programa para su formación, en el área profesional se encuentran las ciencias que argumentan y le dan base a la práctica profesional; los componentes que le permiten a los y las estudiantes reconocer los diferentes contextos y sus individuos se enmarcan en el área complementaria; por último, en el área de praxis investigativa se resaltan los espacios que le faciliten a los y las estudiantes el desarrollo de sus habilidades científicas.

El currículo del programa se divide en tres ejes principales, el primero hace alusión a prácticas y problemas en torno a la sustentabilidad de la agricultura tradicional, la visión empresarial del agro y la exigencia de los mercados por productos limpios y certificados; el segundo eje, se refiere a la investigación, teniendo como objetivo sensibilizar y concientizar a los y las estudiantes acerca de las situaciones actuales referentes a las realidades socioeconómicas de la población del país; y el último eje, resalta la fundamentación en ciencias y áreas del conocimiento en formación social y humanista, agroecología, formación empresarial, química y biología.

El proyecto se ha enfocado en el desarrollo de una educación inclusiva que ofrezca respuestas a las necesidades de las personas y la permanencia en el proceso educativo a través de la formación en proyectos y la ejecución de estos en las comunidades teniendo en cuenta sus dinámicas sociales, económicas y políticas. En relación con el proceso de admisión, la institución no abre inscripciones, sino que va a los territorios en busca de los y las jóvenes de zonas rurales que han sido víctimas del conflicto, "con la ayuda de los Obispos y Párrocos, Rectores y profesores de los colegios, líderes comunales, y organizaciones, se convoca en algún centro educativo o cultural a los jóvenes hombres y mujeres- con perfil de líderes" (Universidad de la Salle, 2015, p. 5).

Por esto, la importancia que se le ha dado a que los y las estudiantes una vez finalicen sus estudios retornen a sus territorios y zonas de origen, para fortalecer y optimizar la calidad de vida de las comunidades y las actividades agrícolas de estas. Según Castrillón (2020),

... jóvenes capaces y motivados de aquellas zonas que han sido afectadas por la violencia, el conflicto, los cultivos ilícitos, los altos índices de pobreza estructural y la falta de oportunidades, se han convertido en líderes que trabajan para transformar social, política y productivamente sus territorios. (párr. 3).

Por otra parte, para Fernández (2020), el programa Utopía "busca en los jóvenes a los futuros líderes del sector rural 
colombiano, hombres y mujeres, las estrategias didácticas que en Utopía se tejen, tienen un valor social especialmente importante porque contribuyen a cerrar brechas de inequidad en la educación" ( $p$. 5). Lo anterior se logra evidenciar en los diferentes espacios formativos para la generación de competencias orientadas al desarrollo rural y a promover la empresarización del campo. Es por esto, que dentro de las características de las y los egresados se encuentra el contar con los conocimientos, metodologías, estrategias, aprendizajes científicos, tecnológicos y de organización campesina para entender y proponer soluciones referentes a los procesos de la agricultura y el desarrollo sustentable.

\section{La Universidad Campesina Asociación para la Promoción Integral de Comunidades Rurales (Asopricor), Uniminuto, Colombia y Algoma, Canadá}

En el 2012, la Uniminuto se contactó con personas de la organización Asopricor, organización que reúne comunidades campesinas de las Regiones del Alto Magdalena y el Tequendama (Cundinamarca) en el centro de Colombia. Después de año y medio de reflexión con las comunidades campesinas se realizó el primer proyecto que fue la sistematización de experiencias pedagógicas, metodologías y saberes ancestrales de las comunidades pertenecientes a Asopricor. (Rivera \& Ariza, 2016, p. 3).

Con base en los saberes reconocidos, las comunidades decidieron crear un programa académico que recogiera el vasto conocimiento de las comunidades. Desde el 2014 la Uniminuto y la Universidad Algoma de Canadá vienen creando el programa Técnico Profesional en Desarrollo Económico, Social y Comunitario. Las y los investigadores para crear la propuesta se plantearon preguntas como estas: ¿Cómo integrar los saberes de las comunidades Asopricor a las universidades Uniminuto y Algoma?, ¿Cómo puede esta integración mejorar los saberes campesinos y universitarios de manera que se beneficien otras comunidades campesinas de Colombia y el mundo?

La experiencia de construcción del sueño de la Universidad campesina de Asopricor se basa en las ideas de Freire (2002), sobre el diálogo de saberes y la importancia de la horizontalidad en las relaciones entre educadores/as y los y las participantes en los procesos educativos, por esto consideran central partir de las capacidades y potencialidades de los y las campesinos y de sus organizaciones.

Las y los investigadores de 
Uniminuto, de la Universidad de Algoma y los miembros de Asopricor fundamentan su propuesta en la pedagogía de los saberes campesinos Núñez (2004), el cual considera fundamental en los procesos educativos rurales retomar los saberes campesinos.

Con respecto a la propuesta curricular, Rivera y Ariza (2016) afirman que con base en los debates con campesinos y campesinas se incluyeron temáticas basadas en sus intereses $\mathrm{y}$ necesidades como personas del campo. Las asignaturas están pensadas para formar un técnico profesional que apoye la organización social, la economía comunitaria campesina. Se incluyen asignaturas relacionadas con la formación en valores, en capacidades organizativas, capacidades de vinculación con las comunidades y capacidades de investigación social. Además, el reconocimiento y lectura de su propia realidad es muy importante en esta propuesta curricular.

Los y las investigadoras y constructoras de la propuesta curricular declaran que las temáticas surgieron con base en largas conversaciones y de este modo se llegaron a acuerdos sobre asignaturas como: "Campesino y Ciudadanía, Nuevas Ruralidades, Cartografía Regional, Desarrollo
Sostenible y Medio Ambiente, entre otras" (Rivera \& Ariza 2016, p. 4).

En el programa se vislumbra la utilización de estrategias metodologías basadas en el diálogo de saberes inspiradas en el pensamiento de Paulo Freire, estrategias metodológicas de trabajo intergeneracional apoyadas en la tradición oral y en el reconocimiento de los saberes campesinos.

El proyecto en su cuarta fase se encuentra desarrollando los talleres intergeneracionales. Este se referencia como "El Circulo" y tiene el propósito de alimentar las asignaturas del técnico profesional mediadas por las experiencias que relatan los y las participantes en los talleres y a su vez, orientar la institucionalización del programa ante el Ministerio de Educación. Así, se busca contribuir en la formación profesional de las y los jóvenes campesinos para que se queden en sus veredas, y proyecten sus sistemas agrícolas a escenarios autosostenibles que no solo cuiden el medio ambiente y sus comunidades, sino que también generen progreso en la región.

Para las y los creadores de esta experiencia son esenciales los procesos de construcción participativa de las propuestas de contenidos, para lograr esto se han tenido en cuenta los rasgos culturales, los sentires y la cosmovisión de 
las comunidades. (Rivera \& Ariza, 2016).

Se destaca de esta experiencia la importancia de recoger los saberes campesinos por medio de la Investigación Acción Participativa, tanto para la construcción de la propuesta curricular como para el desarrollo de sus estrategias metodológicas.

\section{Licenciatura Madre Tierra en la Educación Superior de la Universidad de Antioquia}

Actualmente los pueblos indígenas identifican y canalizan estrategias que han permitido vincularse con la Universidad de Antioquia (UdeA), tal como lo expresa Green (2018), en el año 2000, luego del proceso de reflexión de los indígenas de la región se explicita la necesidad de hacer realidad la educación que tuviese como centro la Madre Tierra, y la UdeA se convierte en la aliada que posibilita el desarrollo de la propuesta.

De acuerdo a ello, la UdeA tuvo como propósito incluir a los pueblos indígenas del departamento de Antioquia, y a partir de la firma de un acuerdo académico se permite la admisión a esta institución de grupos étnicos desde 1983. Ello ha generado un incremento de estudiantes indígenas en la universidad. Así mismo, se logra la creación del Programa de Educación Indígena, adscrito a la Facultad de Educación. En el 2009 se denomina Licenciatura en Pedagogía de la Madre Tierra, insertándose en los procesos académicos y administrativos (Tamayo, 2018).

Esta licenciatura se fundamenta en la idea de que existen otras formas de conocimiento, otros saberes y miradas, se busca que los saberes occidentales que se enseñan en instituciones educativas formales como la universidad se sienten a dialogar respetuosamente con los saberes indígenas. Se evidencia la importancia de retomar como prioridad la construcción del conocimiento comunitario, siendo provechoso los aportes dados por las y los líderes, y sabios de cada pueblo según lo expresa Tamayo (2018), “Con quienes formularon las estrategias para concretar la propuesta de la licenciatura. Se retoma a la tierra, como eje transversal de conocimiento y madre, este último concepto abordado así, las comunidades indígenas tanto de Colombia como de diversos países" (p. 763).

La Licenciatura está estructurada en 3 ciclos de profundización, cada uno de estos ciclos comprende espacios en los que se desarrollan y profundizan los conocimientos y saberes adquiridos, a saber: El ciclo básico se compone de los primeros 4 semestres y abarca los momentos de estudio del Origen y Desequilibrio a partir de espacios de 
formación como el seminario integral, lenguas, plan de vida y proyección comunitaria-práctica pedagógica. Para el ciclo de transición se desarrollan los semestres 5 y 6, que comprenden el momento de construcción de caminos hacia Sanación de las personas con la madre tierra, en este se utilizan espacios de formación como lenguas originarias y castellana, políticas públicas y significados de vida, etc. En el ciclo de profundización se engloban los últimos 4 semestres a partir del momento de la ruta pedagógica denominada Protección, en la cual los y las estudiantes escogen un énfasis de profundización entre: Lenguajes e Interculturalidad; Salud Comunitaria Intercultural y Ordenamiento y Autonomía Territorial (Universidad de Antioquia, s.f.).

Los objetivos de esta propuesta consisten en desarrollar procesos educativos tendientes a la vivificación de las lenguas originarias. Así mismo, contribuir a la aprehensión de aspectos en salud y buen vivir según las tradiciones de los pueblos desde propuestas pedagógicas alternas de acuerdo al territorio que permitan protegerlo y sanarlo.

Su estructura se asemeja en aspectos formales a otros programas de educación de la UdeA. Del primero hasta el sexto semestre los y las estudiantes tienen áreas de núcleo común y de conocimiento base que les permitirán reconocer y comprender su contexto, en donde abordan aspectos de la vida de los pueblos indígenas y su interacción con otros saberes, lenguajes y culturas, a partir del séptimo semestre se da inicio a la profundización lo que permite que los y las estudiantes fortalezcan la gobernabilidad y planes de vida, de igual forma el desarrollo del tejido curricular pretende formar a los y las estudiantes de manera integral desde diferentes encuentros, los regionales con una duración de 12 días, zonales de 10 días, dos veces por semestre; y locales una vez por semestre (Organización Indígena de Antioquia, s.f.).

La construcción del trabajo se hace de manera colectiva,

... en mi equipo cada uno habla y opina sobre los diferentes autores que han escrito sobre los temas, las películas, narraciones, música, teatro que se pueden utilizar, y a partir de allí se elabora el documento que asigna las tareas que debe desarrollar cada uno. (Green, 2018, p. 62).

Los y las profesores son reconocidos como facilitadores y/o acompañantes, los cuales deben tener capacidad de escucha, interpretación de contextos, autoformación permanente, capacidad integración de colectivo, concibiendo la vida comunitaria.

Esta licenciatura, recupera el concepto y las formas de relacionarse con la madre tierra, convirtiéndola en una 
reivindicación para la academia y la cultura. A través de esta propuesta también se busca la cohesión de las comunidades indígenas y consolidar un plan de vida en torno al territorio, a la tierra, que genere iniciativas que contribuyan con el bienestar del colectivo. Por tanto, se resalta la tierra como forma de aprendizaje para todos y todas y no como medio de explotación.

\section{Consideraciones finales: Aportes de las experiencias a la construcción de una propuesta curricular de Educación Superior Rural para el DRTT}

Reconocemos que las experiencias presentadas anteriormente son diferentes entre sí, sin embargo, a manera de síntesis, de ellas podemos obtener una serie de ideas claves para la construcción de una propuesta curricular de educación superior rural para el DRTT.

Como se puede constatar, para construir una propuesta curricular es imperativo reconocer las condiciones históricas, sociales y culturales de la población con la que se quiere trabajar. Las experiencias presentadas son altamente contextualizadas, buscan responder a las necesidades y expectativas de las comunidades.

Para la formulación de una propuesta curricular, consideramos que los principios que la constituyan deben contener elementos sobre: el campo, las y los campesinos, las comunidades indígenas, la ruralidad en el marco de la globalización; la base agroecológica, y la agricultura familiar; los fundamentos epistemológicos, el papel de los saberes campesinos e indígenas, el diálogo de saberes, la justicia epistémica, el reconocimiento de procesos pedagógicos que generen arraigo y sentido de pertenencia, la relevancia de la articulación, trabajo académico y trabajo comunitario, la articulación teoría-práctica (Farias, Alves \& Faleiro, 2020), y los proyectos productivos, entre otros.

Las experiencias se fundamentan en la valoración de los saberes y tradiciones de los y las campesinas e indígenas, considerándolos como sujetos y sujetas culturales (Gómez, 2019). En las experiencias estudiadas, la educación tiene como principio el vincular la educación a los procesos sociales del territorio y, de esta forma, posibilitar que la educación aporte a la transformación social. Otro principio es la formación integral, se busca que la formación no sea solamente técnica, sino también política, social, ecológica, en valores y ciudadana. Así, se resalta la importancia de la conexión entre los asuntos ambientales, sociales y políticos. El trabajo, por su parte, se considera un principio que orienta los procesos educativos, tanto el trabajo productivo, como el trabajo en servicios en espacios 
colectivos. Otro criterio de fundamento hallado en las experiencias es el de la autonomía, el cual debe permitir el desarrollo de metodologías y estructuras curriculares, organizativas basadas en los intereses de los y las estudiantes participantes y de sus comunidades.

De esta forma, las propuestas curriculares son, en gran parte, integrados e interdisciplinarios (articulación de disciplinas y áreas del saber), están organizados en núcleos, ciclos y áreas de formación. Los núcleos básicos o de iniciación de las propuestas estudiadas son de trascendencia, en ellos además de reconstruir los saberes de los campesinos, campesinas, e indígenas y de reconocer sus culturas, se busca desarrollar procesos de formación en lectura, producción de textos, uso de medios informáticos, comprensión del contexto donde se vive y de sus relaciones con la globalización y con las políticas para el campo, al tener en cuenta las políticas globales y los cambios estructurales en los aspectos económicos y sociales (por ejemplo migraciones, crisis climática y ambiental) a nivel mundial y regional de América Latina.

Así mismo, las propuestas curriculares incluyen ejes, actividades o áreas curriculares que amplían la formación específica laboral y donde se fortalecen las capacidades comunicativas de interacción en la comunidad y con otras comunidades. Para la construcción de propuestas de educación para el DRTT es necesario tomar en consideración la cosmovisión indígena, el ser de la tierra y la naturaleza como eje articulador, entonces es central partir del Plan de Vida y la cosmovisión del pueblo Pijao (la comunidad indígena que vive en el DRTT).

Es de resaltar que las experiencias tienen como uno de sus propósitos el formar seres humanos que reconozcan su historia y tengan un sentido de pertenencia con sus territorios, lo anterior se fortalece a partir del trabajo orientado a la solución a los diferentes problemas de sus contextos, además se busca formar ciudadanos y ciudadanas con una perspectiva del cuidado, pues es así como se contribuye a la construcción de una sociedad justa y en paz. Port tanto, las experiencias buscan que las y los participantes sean capaces de analizar los problemas (productivos, sociales y culturales) de sus territorios, proponiendo iniciativas, donde se gestionan los recursos de forma comunitaria y justa.

En cuanto a las estrategias metodológicas, son fundamentales: el aprender de la experiencia, la alternancia entre el estudio en la academia y el aprendizaje en la comunidad, el aprender por medio del trabajo en la comunidad 
(productivo y de servicio a la comunidad).

Son también esenciales, las metodologías basadas en proyectos investigativos, productivos y culturales de acuerdo con los intereses de los y las estudiantes y de las comunidades. Las estrategias metodológicas se basan en el diálogo de la academia con los saberes ancestrales campesinos e indígenas; también reconocen la necesidad de la investigación y en este caso la Investigación Acción Participativa.

Es transversal a las experiencias el reconocimiento de saberes no sólo agropecuarios, sino también organizativos, administrativos de las comunidades y sus organizaciones, lo que nos lleva a pensar en metodologías que propicien el diálogo de saberes y los diálogos intergeneracionales. De las experiencias vemos necesario partir desde la cosmovisión, y los saberes ancestrales de las comunidades indígenas y campesinas, esto supone unas pedagogías interculturales que potencien el aprender de los y las mayores y el fortalecer los saberes sobre el cuidado de la madre tierra.

Así mismo, las experiencias nos proporcionan una mirada colectiva, de autonomía de las y los sujetos y comunidades, rasgos que suponen una articulación de la apuesta pedagógica con movimientos sociales y organizaciones comunitarias.

\section{Referencias}

Acevedo, A. (2013). La agroecología como respuesta a las potencialidades $y$ retos de la agricultura indígena $y$ campesina en el distrito de riego el triángulo del sur del Tolima. Grupo Semillas. Recuperado el 30/07/2021. http://www.semillas.org.co/apc-aafiles/5d99b14191c59782eab3da99d8f9512 6/potencialidades-y-retos-de-la-economaindgena-y-campesina-del-sur-deltolima.pdf

Arias, K. (2016). Hacia una posible conceptualización de la educación intercultural en contextos indígenas. Educadi, 1(2), 59-82.

Bicalho, R. (2017). Historia de la educación del campo en Brasil: El protagonismo de los movimientos sociales. Revista Teias, 18(51), 210-224. https://doi.org/10.12957/teias.2017.24758

Barbosa, L. (2014). Educação do campo, movimentos sociais e a luta pela democratização da educação superior: os desafios da universidade pública no Brasil. Buenos Aires: Clacso.

Caicedo, J., \& Calderón, J. (2016). Currículo: en búsqueda de precisiones conceptuales. Revista Educación y Pensamiento, 23(23), 57-76.

Caldart, R. (2003). A escola do campo em movimento. Currículo sem Fronteiras, 3(1), 60-81.

Calvo, G., \& Castro, Y. (1995). La Familia en Colombia un estado del arte de la investigación 1980-1994. Bogotá: ICBF. 
Castrillón, S. (2020). "Utopía", un proyecto de todos. Recuperado el 04/02/2021.

https://www.elespectador.com/especiales/u topia-un-proyecto-de-todos/

DANE. (2018). Resultados censo nacional de población y vivienda 2018. Bogotá: Departamento Administrativo Nacional de Estadística. Recuperado el 30/07/2021. https://www.dane.gov.co/files/censo2018/i nformacion-tecnica/cnpv-2018presentacion-3ra-entrega.pdf

De Alba, A. (1998). Currículum: crisis, mitos y perspectivas. Buenos Aires: Miño y Dávila Editores. Recuperado el 25/01/2021.

http://www.terras.edu.ar/biblioteca/1/CRR

M_De_Alba_Unidad_1.pdf

DNP Colombia. (2018). Bases del Plan Nacional de Desarrollo 2018-2022, Pacto por Colombia, Pacto por la Equidad. Bogotá: Departamento Nacional de Planeación.

Esteva, G. (2014). La libertad de aprender. Revista Interuniversitaria de Formación del Profesorado, 28(2), 39-50. Recuperado el $04 / 02 / 2021$

https://www.redalyc.org/articulo.oa?id=27 433840005

Ezpeleta, J., \& Weis, E. (1996). Escuelas rurales en zonas de pobreza y sus maestros. Revista Mexicana de Investigación Educativa, 1(1). 53-69. Recuperado el 04/02/2021.

https://www.redalyc.org/articulo.oa?id=14 $\underline{000105}$

Farias, M. N., Alves, M. Z., \& Faleiro, W. (2020). Regime de alternância na Licenciatura em Educação do Campo: uma experiência da Universidade Federal de Goiás/Regional Catalão. Revista Brasileira De Educação Do Campo, 5, https://doi.org/10.20873/uft.rbec.e6192
Fernández, J. (2020). Utopía: una innovación académica para la Colombia rural. Bogotá: Universidad de la Salle, Recuperado el 10/02/2021. https://colab.colombiaaprende.edu.co/expe riencias/utopia-una-innovacion-academicapara-la-colombia-rural/

Flórez, R. (1994). Hacia una Pedagogía del Conocimiento. Revista Educación y Pedagogía, (12 y 13), 339-350.

Freire, P. (2002). Pedagogía del oprimido. México: siglo XXI

Freitas, L. (2010). A escola única do trabalho: explorando os caminhos de sua construção. Cadernos do ITERRA, 1(15), 155-175.

García, J., \& Cañal, P. (1995). ¿Cómo enseñar? Hacia una definición de las estrategias de enseñanza por investigación. Investigación en la escuela, 25, 5-16.

Gómez, E. (2019). Memoria biocultural, saberes locales agrícolas de los contextos locales integrados en la educación media técnica agropecuaria. Revista Arbitrada del CIEG, 39, 82-91.

Gómez, M., Galeano, C., \& Jaramillo, D. A. (2015). El estado del arte: una metodología de investigación. Revista Colombiana de Ciencias Sociales, 6(2), 423-442.

https://doi.org/10.21501/22161201.1469

Green, A. (2018). Pedagogía de la Madre Tierra: "Un deber histórico de nosotros los humanos" Entrevista realizada por investigadores Universidad de Cundinamarca. Caminos Educativos, 5(1), 59-67. Recuperado el 02/02/2021. https://www.ucundinamarca.edu.co/docum ents/comunicaciones/revistacaminos/caminos-educativos-5.pdf

Jiménez, A. (2004). El estado del arte en la investigación en las ciencias sociales. 
En: La práctica investigativa en ciencias sociales. Bogotá: UPN. Recuperado el 28/07/2021.

http://bibliotecavirtual.clacso.org.ar/Colom bia/dcs-upn/20121130050742/estado.pdf

Londoño, P., \& Calvache, J. (2010). Las estrategias de enseñanza: aproximación teórico-conceptual. En Vásquez Rodríguez, F. (Ed.). Estrategias de enseñanza Investigaciones sobre didáctica en instituciones educativas de la ciudad de Pasto (pp. 11-32). Bogotá: Universidad de la Salle e Institución Educativa CESMAG. Recuperado el 25/02/2021. http://biblioteca.clacso.edu.ar/Colombia/fc e-unisalle/20170117011106/Estrategias.pdf

Lopes, A., \& Macedo, E. (2011). Teorías de Currículo. Cadernos de Pesquisa, 4 (150), 1076-1081. Recuperado el 25/01/2021.

https://dialnet.unirioja.es/servlet/articulo?c $\underline{\text { odigo }=6209399}$

Magendzo, A. (1986). Curriculum y Cultura en América Latina. Santiago: Programa Interdisciplinario de Investigaciones en Educación. Recuperado el 25/01/2021. http://www.piie.cl/biblioteca/publicaciones /libros/L-1986-044.pdf

Molina, M., \& Sá, L. (2015). A educação como lugar de disputa e resistência: registros e reflexões sobre uma experiência. Formação de educadores do campo no Brasil. En Medina, P. (Ed.). Pedagogías insumisas Movimientos político-pedagógicos y memorias colectivas de educaciones otras en América Latina (pp. 129-160). México: Universidad de Ciencias y Artes de Chiapas Centro de estudios superiores de México y Centroamérica educación para las ciencias en Chiapas, A.C. Juan Pablos editor. Recuperado el 25/01/2021. https://repositorio.cesmeca.mx/bitstream/h andle/11595/847/L\%202015\%20-
\%20Pedagog\%c3\%adas\%20insumisas.pdf ?sequence $=7 \&$ is Allowed $=y$

Núñez, J. (2004). Los saberes campesinos: Implicaciones para una educación rural. Investigación y Postgrado, 19(2), 13-60.

Organización Indígena de Antioquia. (s.f). Licenciatura en pedagogía de la madre tierra. Antioquía. Recuperado el 04/02/2021,

http://www.enlazandoculturas.cicbata.org/s ites/default/files/Licenciatura\%20en\%20Pe dagog\%C3\%ADa\%20de\%20la\%20Madre \%20Tierra..pdf

Oster-Katz, L. (2005). Aprender sin Educación: El Aprendizaje, la Historia Personal y el Contexto Comunitario en la Experiencia de la Universidad de la Tierra. Independent Study Project (ISP) Collection. 439. Recuperado el 04/02/2021.

https://digitalcollections.sit.edu/isp_collect ion/439/

Páramo, P. (2011). La investigación en ciencias sociales (Primera). Bogotá: Universidad Piloto de Colombia.

Pineda, M., \& Suaza, D. (2017). Una reflexión crítica de la educación rural desde la experiencia propia, hacia un proceso que promueva la resignificación de la identidad campesina y el arraigo al territorio. Universidad de Antioquia, Facultad de Educación Maestría en Educación línea pedagogía y diversidad cultural cohorte especial Madre Tierra. Recuperado el 02/02/2021. https://bibliotecadigital.udea.edu.co/bitstre am/10495/10063/1/PinedaMaria_2018_edu cacionrural.pdf

Poder Legislativo, Colombia: El Acuerdo Final de paz. La oportunidad para construir paz. (Cartilla completa del Acuerdo). Junio 2016, Recuperado el 25/01/2021. https://www.refworld.org.es/docid/5a874f2 54.html 
Rivera, M., \& Ariza, C. (2016). Tras el sueño de la universidad campesina, Asopricor - Colombia. Ponencia en el V Encuentro Latinoamericano de Metodología de las Ciencias Sociales (ELMeCS). Métodos, metodologías y nuevas epistemologías en las ciencias sociales: desafíos para el conocimiento profundo de Nuestra América. Mendoza: FCPYS-UNCUYO. Recuperado el 21/12/2020. http://elmecs.fahce.unlp.edu.ar

Cundinamarca.gov.co. (2017). Recuperado el 25/01/2021. http://www.cundinamarca.gov.co/Home/Se cretariasEntidades.gc/Secretariadeagricultu ra/Secagriculturadespliegue/asdesarrollorur al_contenidos/csecreagri_distritos-de-riego

Serna, J., \& Patiño, S. (2018). Educación y desarrollo humano en los contextos rurales. Revista Temas III (12), 189-200. https://doi.org/10.15332/rt.v0i12.2042

Silva, T. (2006). O currículo como fetiche. A poética e a política do texto curricular. Belo Horizonte: Autêntica.

Tamayo, C. (2018). Licenciatura en Pedagogía de la Madre Tierra, etnomatemática y formación de profesores. Ciência \& Educação, 24(3), 759-777. https://doi.org/10.1590/1516$\underline{731320180030014}$

Universidad de Antioquia (s.f). Plan de estudios. Recuperado el 02/02/2021, http://www.udea.edu.co/wps/portal/udea/w eb/inicio/unidades-

academicas/educacion/oferta-

pregrado/licenciatura-pedagogia-madre-

tierra/contenido/asmenulateral/plan-de$\underline{\text { estudios }}$

Universidad de la Salle. (2015). Utopía. Documento PDF.

Universidad de la Salle. (2020). Utopía. Recuperado el 10/02/2021. https://www.utopia.edu.co/wcm/connect/5

03e94a9-55b7-4c65-ab7ad35f91c48884/Caso+Proyecto+Utop\%C3 \%ADa+2020.pdf?MOD=AJPERES\&CVI $\mathrm{D}=$ noMwlaE

Zaldivar, J. (2009). La Universidad de la Tierra en México. Una propuesta de aprendizaje convivencial. Temas $y$ perspectivas sobre educación. La infancia ayer y hoy (Globalia). Recuperado el 04/04/2021.

https://www.academia.edu/4699834/La_U niversidad_de_la_Tierra_en_M\%C3\%A9x ico_Una_propuesta_de_aprendizaje_convi vencial?auto=download

\footnotetext{
${ }^{\mathrm{i}}$ Un distrito de riego es un área de influencia de las obras de infraestructura destinadas a dotar a la misma con riego, drenaje o protección contra inundaciones, con el propósito de elevar la productividad agropecuaria. (Secretaria de Agricultura de Cundinamarca, 2017, Párr. 1).
}

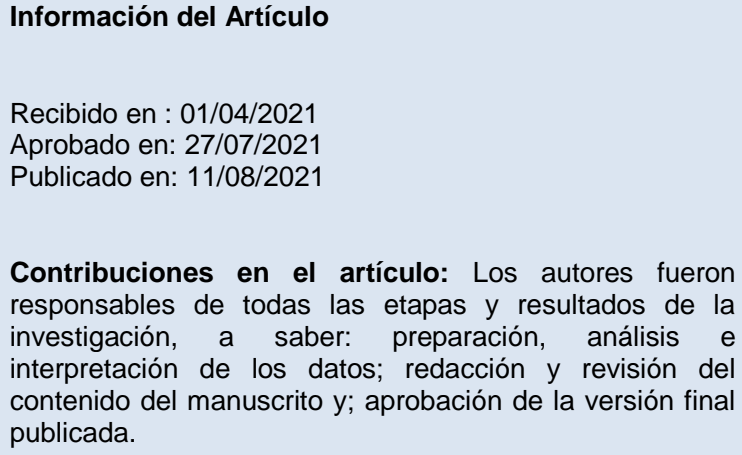
responsables de todas las etapas y resultados de la investigación, a saber: preparación, análisis e interpretación de los datos; redacción y revisión del contenido del manuscrito y; aprobación de la versión final publicada.

Conflictos de Intereses: Los autores han declarado que no existe conflicto de intereses con respecto a este artículo.

Evaluación del artículo

Revisión por pares

Agencia de Desarrollo

Sistema Universitario Estatal. 


\section{Cómo citar este artículo}

\section{APA}

Ochoa, L. V. A., Salamanca, L. M. G., Escobar, J. J. P., \& Cuervo, L. F. R. (2021). Aportes de experiencias de Educación Superior Rural de Colombia, Brasil y México a la construcción de una propuesta curricular en el Sur del Tolima (Colombia). Rev. Bras. Educ. Camp., 6, e11934. http://dx.doi.org/10.20873/uft.rbec.e11934

\section{ABNT}

OCHOA, L. V. A.; SALAMANCA, L. M. G.; ESCOBAR, J. J. P.; CUERVO, L. F. R. Aportes de experiencias de Educación Superior Rural de Colombia, Brasil y México a la construcción de una propuesta curricular en el Sur del Tolima (Colombia). Rev. Bras. Educ. Camp., Tocantinópolis, v. 6, e11934, 2021. http://dx.doi.org/10.20873/uft.rbec.e11934 